\title{
Design of high scalability multi-subcarrier RoF hybrid system based on optical CDMA/TDM
}

\author{
Ahmed Ghanim Wadday, Faris Mohammed Ali, Hayder Jawad Mohammed Albattat \\ Department of Communications Techniques Engineering, Engineering Technical College, Al-Furat Al-Awsat Technical \\ University, Al-Najaf, Iraq
}

\begin{abstract}
Article Info
Article history:

Received Jun 22, 2020

Revised Aug 23, 2020

Accepted Sep 7, 2020

Keywords:

Hybrid optical system

OCDMA/OTDM

Scalability hybrid system

ABSTRACT

The technology of radio over fiber ( $\mathrm{RoF}$ ) regard a crucial point to solve problems in wireless communication system. As well as, the growth of internet applications also reveals a tremendous increase in bandwidth for different applications. Therefore, the development of optical networks is very important that have maximum bandwidth by using different multiple access techniques. Optical code division multiple access (OCDMA) technique has considered as a good solution for high bandwidth network. Hybrid optical systems of OCDMA and time division multiplexing (OTDM) has been proposed in this paper to increase the number of simultaneous users. The results of hybrid OCDMA and OTDM system demonstrate that this system can make a considerable increase in the network scalability while ensuring sufficient data rate and an acceptable bit error rate. Where M-user OCDMA signals can be transmitted in different channels of an OTDM system. Due to its wide band facility compared with other access techniques, OCDMA used here. In addition to its high scalability for our radio network, the OTDM and SCM utilized. The combination of these efficient access technique and powerful time-sharing media are lead to increase the framework system scalability
\end{abstract}

This is an open access article under the CC BY-SA license.

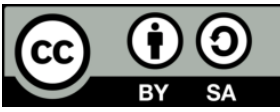

Corresponding Author:

Faris Mohammed Ali

Department of Communications Techniques Engineering

Engineering Technical College

Al-Furat Al-Awsat Technical University, Al-Najaf 31001, Iraq

Email: faris@atu.edu.iq,

\section{INTRODUCTION}

In recent years, the development of optical and wireless communications, radio over optical fiber (RoF) techniques have been regarded a tremendous change in the volume of data flow in wireless access networks and optical communication networks technology, where it considered as the strong solution for future high speed because it have high capacity and bandwidth [1-2]. Moreover, the ability of the RoF system to secure transmissions from being monitored is another major constraint that must be fulfilled in the current network. By using newer coding schemes or to transform the signal to have an elevated level of security are part of struggles that researchers are putting toward making the mobile communication safer and more reliable [3].

Chen C. et al. have illustrated a novel of RoF system to get the better performance. They show that it can transmitted (downstream) 10-Gb/s data over $30 \mathrm{~km}$ using single mode optical fiber to generate and detect a 60-GHz MMW signal with bit-error-rate (BER) reach to 10-9 after the transmission over 30- $\mathrm{km}$ single mode optical fiber [4]. 
The numerical analyzed study for the differential phase OCDMA system based on RoF has conducted both broadcasts and facilities. Here codec (coder/decoder) unit has organized with arrayed waveguide grating (AWG) switches based on complementary Walsh Hadamard (CWH) sequences assigned as signature codes. In such system, it requires two AWG switches to realize radio encoded spectrum of both base and control decoding stations. The performance analyses of this system network was evaluated that take in the account the noise of phase induced intensity noise (PIIN) of OCDMA spectra. Using of this technique that lead to satisfy systems without interference as well as minimized crosstalk noise [5]. Various studies on OCDMA-RoF system was achieved, which can be enhanced by dividing framework of the system into two main substructures central and base stations. By using a fiber optic link to central station with a few distant streamlined base stations, it can essentially raise the system versatility, scope, limit and besides serve both altered and mobile end users. Where, OCDMA-RoF is a favorable multiple access method for high security access system. It combines a high bandwidth of fiber optic with the flexibility of CDMA technique, so it can accomplish high-speed connectivity and guarantee the secure against interference along the fiber optic link. [6-9]. Further, researchers have used RoF Transmission System for 5G Applications, by utilizing non-return to zero (NRZ)-return to zero (RZ) encoding scheme format modulated over optical system carrier of 1550 $\mathrm{nm}$, with using of Mach-Zehnder (MZ) optical system modulator that represented an electrical-optical signal converter. Where RZ encoding format performs better up to the link range of $45 \mathrm{~km}$ of a fiber optic, but as the fiber optic transmission distance increases beyond $45 \mathrm{~km}$, NRZ-encoding scheme is prefer due to better performs than RZ-encoding format [10].

The executing Flexible Cross Correlation (FCC) OCDMA sequence which depend on RoF framework technique to enhance bandwidth and temporarily enhance the data bit rate performance while reduce the impassive optical devices have proposed by some researcher. The FCC-OCDMA technique can effectively minimize the results of PIIN, which lead to cancellation Multiple Access Interference (MAI) that return to the RoF framework, where this system has a simple and easy code generation [11]. In recent years, several other approaches used to scale up the subscriber number, some of them depending on all-optical networks by using a combination of two optical multiplexing techniques. One of those scalability-improving technologies is OCDMA/OFDMA. Another combination has used to carry different data types by used hybrid OCDMA/WDM system [10, 11]. Hybrid subcarrier multiplexing (SCM) combined with spectra amplitude coding SAC-OCDMA technology have investigated for enhancing the networks of RoF framework and achieve a high bandwidth and data rate [12-14]. K. S. Alaoui et al. in 2019 have used the hybrid OFDM / SAC OCDMA approach to implement a ROF relation. ROF technology uses Multi-Carrier Modulation, such as OFDM, which provides the ability to increase bandwidth along with an reasonable amount of costs, and this concept has become an adequate subject recently for several research projects. At the other hand, the SAC-OCDMA method is able to boost device data levels and maximize user numbers, and is the spectral amplitude coding optic code division multiple access method [15]. In 2019 Hybrid TDM / WDM Gigabit Passive Optical network experimental research is carried out by replacement of Free Space Optical channel with the ultimate mile optical fibre. This work demonstrates the efficiency and quality of the FActor and the bit rate of error along with the eye patterns of the hybrid TDM / WDM GPON system using an FSO channel. This network is a complete $2.5 \mathrm{~Gb} / \mathrm{s}$ downstream network and $1.244 \mathrm{~Gb} / \mathrm{s}$ upstream transmission. This system has two directions. To evaluate network performance, the program uses different FSO channel lengths [16].

N. Alsowaidi implemented a cost efficient hybrid configuration based on the multiple access optical code division (CDMA) overlaid WDM grid. Hybrid systems were used to generate 120 user data with a spectral efficiency of $1.6 \mathrm{~b} / \mathrm{s} / \mathrm{Hz}$ with only 15 wavelengths with channel separations of up to $25 \mathrm{GHz}$. In other words, some users can share an optical CDMA technique with one wavelength, at a line speed of 40 Gbps per wavelength. It will increase the quality of the usage of wavelengths. Results show that the hybrid network serves 120 users over $90 \mathrm{~km}$ of single-mode fiber and $15.075 \mathrm{~km}$ of fiber dispersion [17].

New approaches for increasing network capacity are being implemented, including optical code division multiple-access (OCDMA), which is based on an acknowledgement of code for each device via the optical channel. Nonetheless, this technique enables the transmission of different information on the same frequency range, compared to different users, without interfering. OCDMA can also be used for SMF fiber [18], but is not appropriate for MMF because it calls for the insertion, at revieve for the modal scatter of the MMF, of additional optoelectronic components for electrical treatment. Bragg fiber gratings (FBGs), for example, are not consistent with multi-mode fibers when using spectral encoding CDMA [19]. On the contrary, the mode group division multiplexing (MGDM) technique can easily create multiplexes by placing a number of SMFs at many different offsets and then exciting multimode fiber (MG) mode groups [20, 21]. By transportation of baseband signal and radio signals (to $2.5 \mathrm{GHz}$ ), respectively at a dates of 2,5 $\mathrm{Gb} / \mathrm{s}$, the symbolic errors rate (SER) of 3 / 3 MGDM network over MMF fibers is reached below $10^{-10}$ and $10^{-9}$ [22]. For the first time, Mahdi Kasmi et al. have suggested and presented for $5 \mathrm{G}$ communications at a data rate of 
$10 \mathrm{~Gb} / \mathrm{s}$ with the waveform GFDM multiplexing via multimode fibre. The MMF fiber is simulated and measured for different Offsets based on the offset launch technique. Thus, using the GFDM technique, we minimize radiation outside of band (OOB) by $5 \mathrm{~dB}$ compared to OFDM, and achieve an even lower bit-error rate (BER). They further show that by increasing the Volterra equalizer taps we can achieve reasonable BER efficiency with varying offset values [23].

In this work, a multi-subcarrier RoF hybrid system model is based on combining optical CDMA/TDM has been conducted in order to increase scalability of the framework system. The reason behind utilization of OCDMA system technique that is because it has high bandwidth compared with other access techniques, as well, the usage of OTDM and SCM for increasing the scalability of RoF networks.

\section{SYSTEM DESIGN}

In this article, hybrid RoF based on SCM/OCDMA/OTDM has been proposed to increase the scalability and the bandwidth of the optical network. Where this system has used multi-diagonal (MD) code as an OCDMA. However, to evaluate the value of the signal to noise power ratio (SNR) of a system and the bit error rate (BER) have conducted for an OCDMA subsystem. Gaussian approximation is used to calculate $\mathrm{BER}$, where $\sigma 2$ variance of the optical detector, $\sigma \mathrm{b}$ beat noise, and $\sigma$ th thermal noise [24].

$$
\begin{aligned}
& S N R=I^{2} / \sigma^{2} \\
& \sigma^{2}=\sigma_{b}+\sigma_{t h}=2 e B I+\frac{4 K_{b} T_{n} B}{R_{L}}
\end{aligned}
$$

Where B is electrical bandwidth, Tn is receiver noise temperature, $\mathrm{Kb}$ is Boltzmann's constant, RL is receiver load resistance, I is mean of optic current and e is electron load. To the photocurrent $I$. First, let $C_{k}(i)$ and $C_{I}(i)$ are numbers of $\mathrm{k}_{\mathrm{th}}$ sequence of matrix of a code for the encoders and decoders, where direct detection technique is used for a code properties and they are expressed as [24]:

$$
\sum_{i=1}^{L} C_{k}(i) C_{I}(i)=\left\{\begin{array}{cc}
W, & \text { for } k=1 \\
0, & \text { Otherwise }
\end{array}\right.
$$

And the power density of the received optic signal is given as follows:

$$
G(v)=\frac{P_{s r}}{\Delta v} \sum_{k=1}^{K} d_{k} \sum_{i=1}^{L} C_{k}(i) C_{I}(i) \Pi(i)
$$

(Where $K$ is the number of users, $d_{\mathrm{k}}$ is data bit of $k_{\mathrm{th}}$ user, $P_{s r}$ is the effective received power of broadband source, $\Delta v$ is the optical source bandwidth and $\Pi(i)$ given as [16]:

$$
\Pi(i)=u\left[v-v_{0}-\frac{\Delta v}{2 L}(-L+2 i)\right]-u\left[v-v_{0}-\frac{\Delta v}{2 L}(-L+2 i+2)\right]
$$

Where $u(v)= \begin{cases}1 & v \geq 0 \\ 0 & v<0\end{cases}$

To calculate $G(v)$ integral, w will consider an example of the power spectral density (PSD) first, as it is shown in Figure 1, where $A(i)$ is spectrum signal amplitude with width of $\Delta v / L$ :

$$
\begin{aligned}
& \int_{0}^{\infty} G(v) d v=\int_{0}^{\infty}\left[\frac{P_{s r}}{\Delta v} \sum_{k=1}^{K} d_{k} \sum_{i=1}^{L} C_{k}(i) C_{I}(i) \Pi(i)\right] d v \\
& \int_{0}^{\infty} G(v) d v=\frac{P_{s r}}{\Delta v} \int_{0}^{\infty}\left[\sum_{k=1}^{K} d_{k} \cdot W \cdot \frac{\Delta v}{L}\right] d v
\end{aligned}
$$




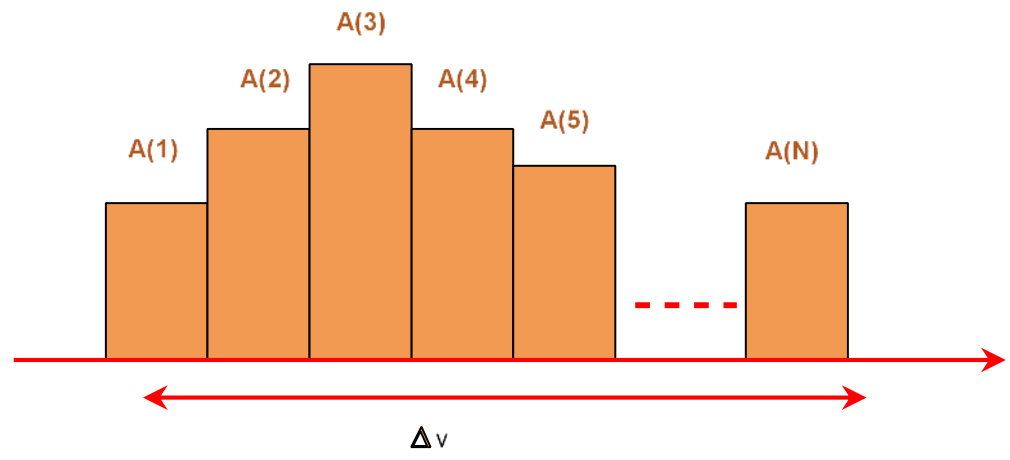

Figure 1 . The power spectral density of the received optic signal

When all users send a bit simultaneously $\sum_{k=1}^{K} d_{k}=d_{1}+d_{2}+\cdots+d_{K}=W$, so

$$
\int_{0}^{\infty} G(v) d v=\frac{P_{s r} W^{2}}{L}
$$

The photocurrent $I$ is given as:

$$
I=\Re \int_{0}^{\infty} G(v) d v
$$

Where $\Re$ is the photodiode responsively, which can be given by $\Re=\eta e / h v_{c}$, and $\eta$ is the quantum efficiency, $v_{c}$ is the optical pulse central frequency of the original broad-band and $h$ is Planck's constant [25]. As shown in (9) can be rewritten as:

$$
I=\Re \int_{0}^{\infty} G(v) d v=\frac{\Re P_{s r} W^{2}}{L}
$$

Substitute (10) into (2):

$$
\sigma^{2}=\frac{2 e B \Re P_{s r} W^{2}}{L}+\frac{4 K_{b} T_{n} B}{R_{L}}
$$

As shown in (11) can be written as follows if the transmitting probability of 0.5 is taken into account for each bit 1 at any time per user:

$$
\sigma^{2}=\frac{e B \Re P_{s r} W^{2}}{L}+\frac{4 K_{b} T_{n} B}{R_{L}}
$$

Hence, SNR and BER of the direct detection technique is expressed as [25]:

$$
\begin{aligned}
& S N R=\frac{I^{2}}{\sigma^{2}}=\frac{\left(\frac{\Re P_{s r} W^{2}}{L}\right)^{2}}{\frac{e B \Re P_{s r} W^{2}}{L}+\frac{4 K_{b} T_{n} B}{R_{L}}} \\
& B E R=\frac{1}{2} \operatorname{erfc}\left(\sqrt{\frac{\left(\frac{\Re P_{s r} W^{2}}{L}\right)^{2}}{\frac{8 e B \Re P_{s r} W^{2}}{L}+\frac{32 K_{b} T_{n} B}{R_{L}}}}\right)
\end{aligned}
$$

Where erfc is the function of complementary error, $\mathrm{L}$ is the code length, $P_{S r}$ is the effective received power of broadband source, $\mathrm{W}$ is the weight which is the number of ones in each row and $\mathfrak{R}$ is the photodiode responsively. This responsively can be given by $\Re=\eta e / h v_{c}$, where $\eta$ is the quantum efficiency, vc is the optical pulse central frequency of the original broad-band and h is Planck's constant. 


\subsection{Multi-diagonal code}

The Multi-diagonal (MD) code is categorized by the $\left(\mathrm{L}, \mathrm{W}, \lambda_{c}\right)\left(\lambda_{c}\right.$ is the in-phase cross correlation) parameters, to construct a diagonal matrix of size $(\mathrm{K} \times \mathrm{L})$. Assume $\mathrm{Ik}$ the identity matrix $(\mathrm{K} \times \mathrm{K})$ :

$$
I_{1}=[1], I_{2}=\left[\begin{array}{ll}
1 & 0 \\
0 & 1
\end{array}\right], I_{3}=\left[\begin{array}{lll}
1 & 0 & 0 \\
0 & 1 & 0 \\
0 & 0 & 1
\end{array}\right], \ldots, I_{K}=\left[\begin{array}{ccc}
1 & \cdots & 0 \\
\vdots & \ddots & \vdots \\
0 & \cdots & 1
\end{array}\right]
$$

By using cross-correlation equation $\lambda_{c}=\sum_{i=1}^{L} X_{i} Y_{i}$ between any two rows of IK can get zero value, which is used to solve the MAI problem and terminate the influence of the PIIN, which are considered the major causes of the SNR limitation. Where $\mathrm{X}=(\mathrm{x} 1, \mathrm{x} 2, \ldots, \mathrm{xL})$ and $\mathrm{Y}=(\mathrm{y} 1, \mathrm{y} 2, \ldots, \mathrm{yL})$ are the two code sequences.

The MD matrix has constructed from the sequences repetition of a diagonal matrix, where $\mathrm{w}$ represented number of repetition. For example of an MD matrix with six users and weight is two, since $\mathrm{K}=4$ and $\mathrm{W}=2$ the length of the MD code is given by $\mathrm{L}=\mathrm{K} \times \mathrm{W}=8$ and codeword by:

$$
\begin{aligned}
& \mathrm{MD}_{\text {code }}=\left[\begin{array}{llllllll}
1 & 0 & 0 & 0 & 1 & 0 & 0 & 0 \\
0 & 1 & 0 & 0 & 0 & 1 & 0 & 0 \\
0 & 0 & 1 & 0 & 0 & 0 & 1 & 0 \\
0 & 0 & 0 & 1 & 0 & 0 & 0 & 1
\end{array}\right]_{4 \times 8} \\
& \text { Optical CDMA codewords }=\left\{\begin{array}{lll}
\operatorname{code} 1 & \Rightarrow & \lambda_{1}, \lambda_{5} \\
\operatorname{code} 2 & \Rightarrow & \lambda_{2}, \lambda_{6} \\
\operatorname{code} 3 & \Rightarrow & \lambda_{3}, \lambda_{7} \\
\operatorname{code} 4 & \Rightarrow & \lambda_{4}, \lambda_{8}
\end{array}\right.
\end{aligned}
$$

\subsection{SCM/OCDMA/OTDM architecture system}

The purpose of a new proposed architecture and scheme system that based on SCM/OCDMA/OTDM to enhance the scalability, bandwidth, and increase the number of simultaneous users. Figure 2 shows the design of a highly scalable hybrid RoF SCM/OCDMA/OTDM framework system with 10 Gbps of data rate. In this architecture system each OCDMA group consist of M codes, where each code has assigned for a group of $\mathrm{X}$ subcarrier signals. After coding process the signals will grouped together by an optical power combiner, and multiplexed with other groups by an optical time division multiplexer to implement $\mathrm{N}$ channels (time slots) of OTDM network, each channel will carry out a particular OCDMA group, as illustrated in Figure 3. Each OTDM transmitter is able to transmit data by a fixed period (tCh). This approach allows the reusing of the same OCDMA codes by other groups in different time slots and allows N groups of OCDMA to transmit data over OTDM system with reusing of the same OCDMA and SCM hardware in all groups. The scalability of this hybrid system depends on the reuse factor, which it is the number of the OTDM channel $(\mathrm{N})$ where larger $\mathrm{N}$ leads to more users and less bandwidth per user. Comparing with only OCDMA system, the new proposed hybrid system provides an increasing in the total number of users by $\mathrm{N}$ times. The maximum simultaneous users (U) of this hybrid SCM/OCDMA/OTDM system can be expressed as(18):

$$
U=X \times M \times N
$$

At the receiving side, to extract the information of a particular user, the time division de-multiplexed (TDM DeMux) will extract the channel that includes the signal of this user then it passed through decoding process of the OCDMA system. After converting this light to an electrical signal, a certain frequency that carry the information of this user will be pass only through a band pass filter (BPF).

Figure 4 is the simulation design of the RoF over SCM/OCDMA/OTDM system with four SCM radio signals, four OCDMA groups and six OTDM channels. Each SCM group consist of four radio transmitters, the output signals are coded by MD OCDMA with $\mathrm{W}=2$ as this clear in the transmitter and receiver subsystems Figures 5 and 6.

The data of each user will be generated by pseudo random bit sequence (PRBS) and using nonreturn to zero (NRZ) pulse generator then an electrical Amplitude Modulator (AM) will be used as an analog amplitude modulator with certain radio frequency. An electrical combiner will combine four multi-subcarrier signals. The electrical modulated signals will be optically modulated by an external optical modulator (Mach-Zehnder modulator) on a light into code sequence at the transmitter end. The Mach-Zehnder which based on an interferometeric principle acts as an intensity modulator. The light source that utilized in the proposed system is a white light source, which has broad optical bandwidth. Two fiber Bragg gratings (DBGs) have used to encode the light as amplitude-spectrally code OCDMA according to a certain code.

Design of High Scalability Multi-Subcarrier RoF Hybrid System Based on ... (Ahmed Ghanim Wadday) 
Finally, all OCDMA modulated code sequences will combined by an optical power combiner and transmitted through the channel. The utilized channel consists of standard single mode fiber (SSMF), dispersion compensating fiber (DCF) and erbium-doped fiber amplifier (EDFA) taking into account the effects of attenuation, dispersion and nonlinearities. At the receiving side, the optical signal passes through an optical TDM demultiplexer then by a splitter into two FBG filters that act as an OCDMA decoder, where direct detection has been used for MD decoding as shown in Figure 6. A PIN photodetector (PIN PD) (with dark current $5 \mathrm{nA}$, responsivity of $1 \mathrm{~A} / \mathrm{W}$ and thermal noise $1.8 \mathrm{e}-024 \mathrm{~W} / \mathrm{Hz}$ ) has been used to convert the optical signal to an electrical signal. Finally, the electrical signal will be split to four signals then each one will passed through a band-pass filter (BPF) with specific frequency and bandwidth, then an electrical amplifier and an electrical amplitude demodulation (ADeM) will be used to retrieve the original information.

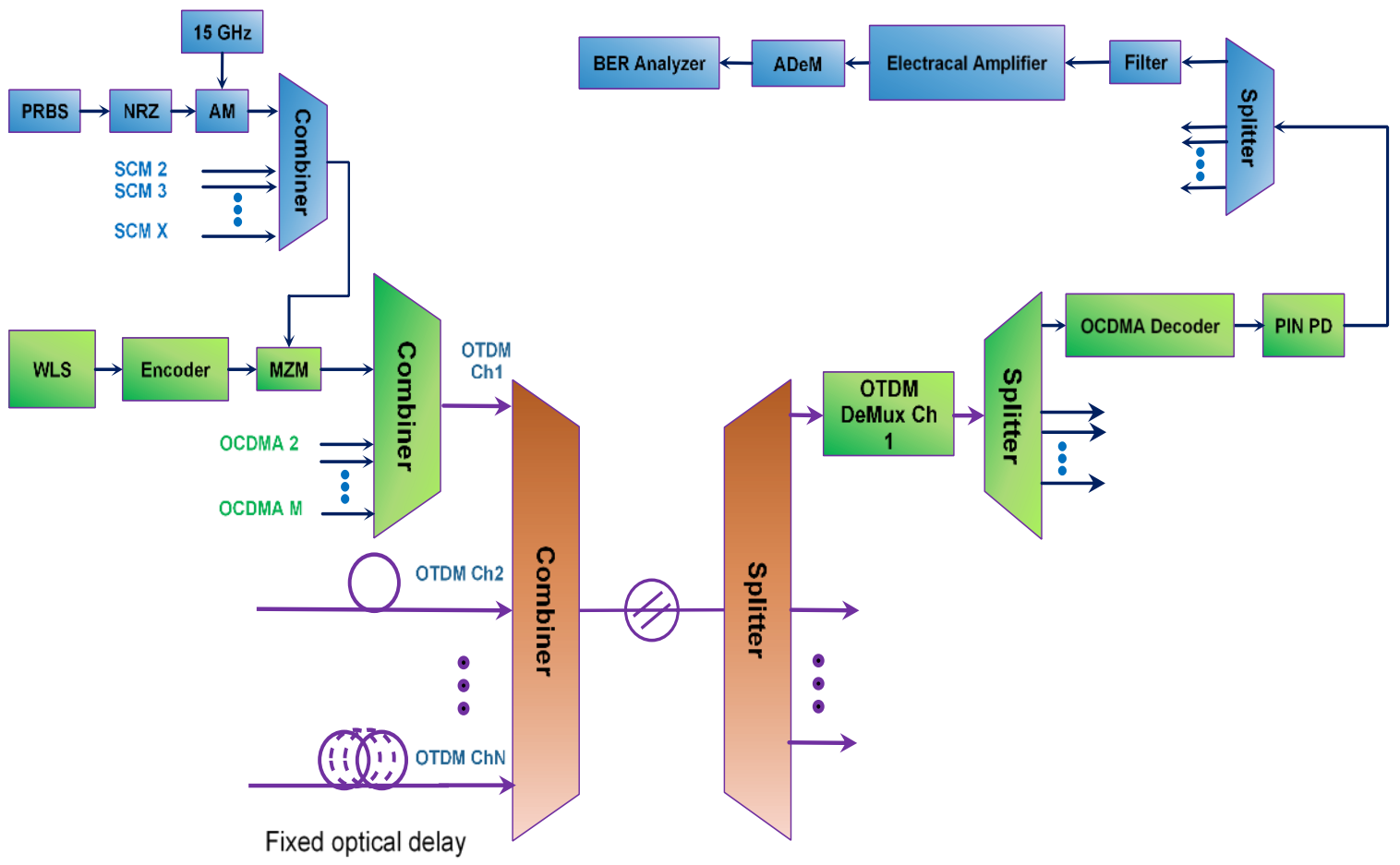

Figure 2. SCM/OCDMA/OTDM hybrid system

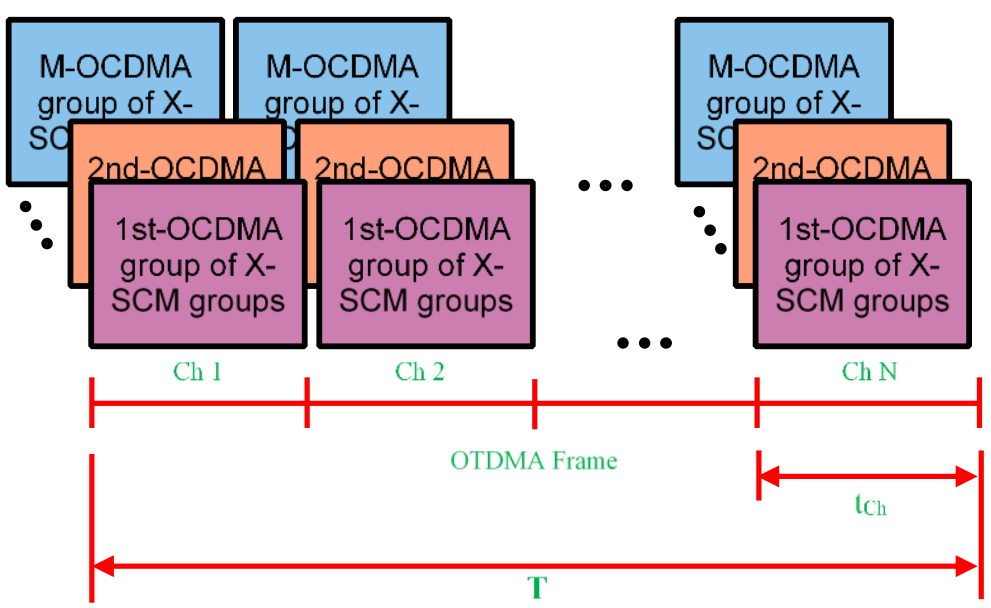

Figure 3. M-OCDMA groups into N-channel of OTDM frame 


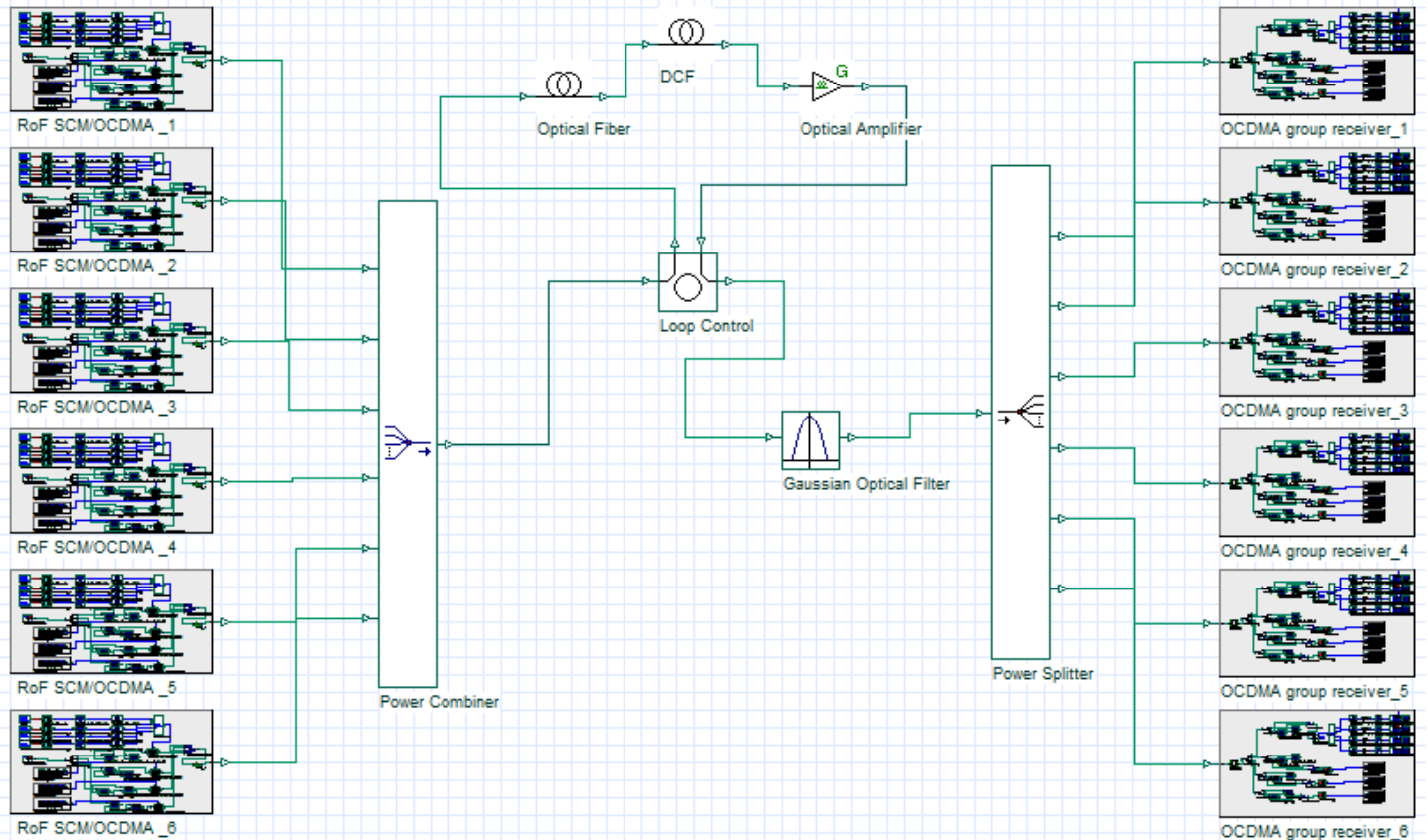

Figure 4. The schematic block diagram of RoF SCM over optical CDMA/TDM hybrid system of 4 OCDMA groups and 6 OTDM channels

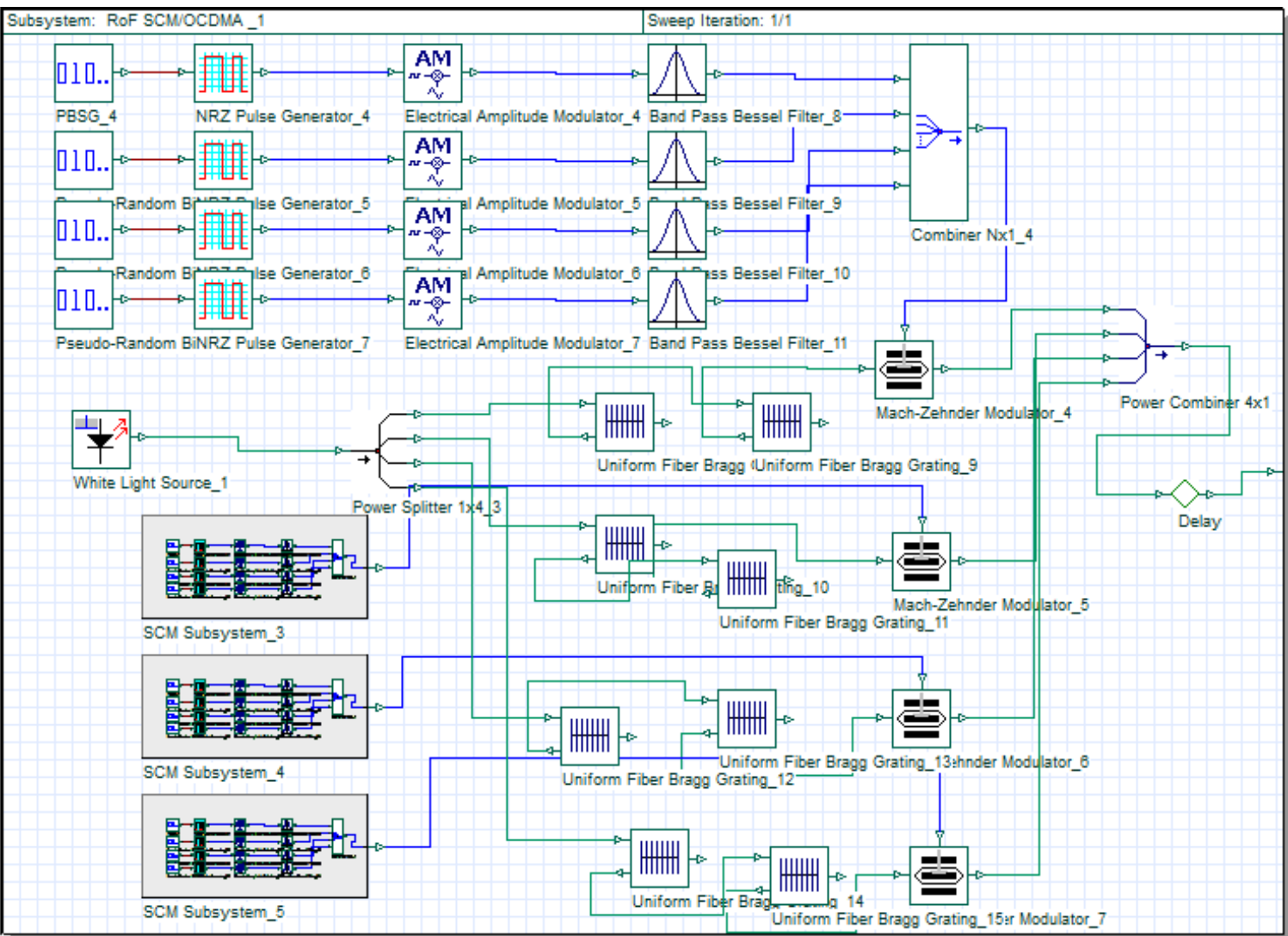

Figure 5. An OCDMA group transmitter subsystem with 4 OCDMA double weight MD encoders each one has 4 subcarrier signals 


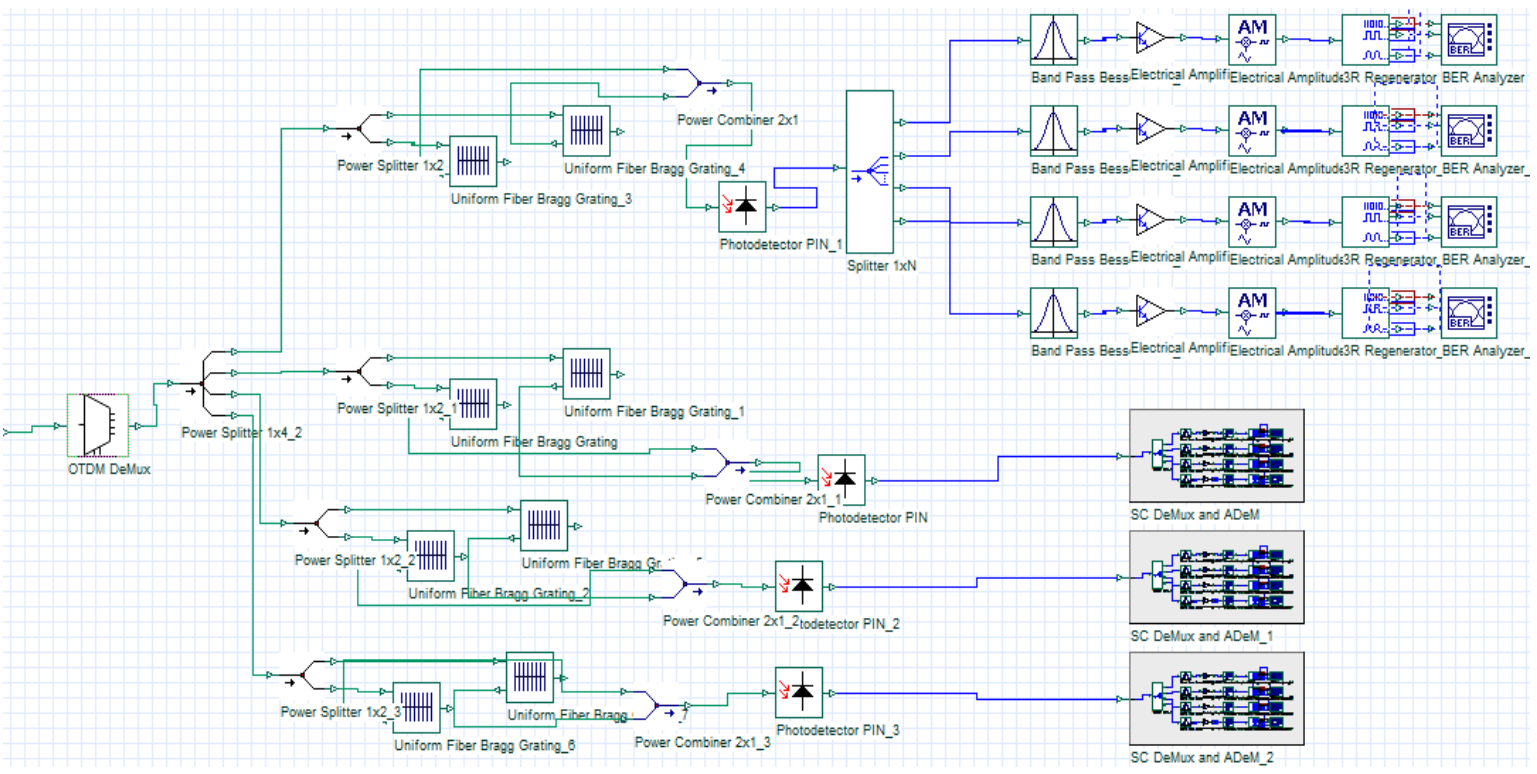

Figure 6. An OCDMA group receiver subsystem of 4 double weight MD-OCDMA decoder, each OCDMA signal feed a group of 4 subcarrier demultiplexers

\section{PERFORMANCE ANALYSIS OF HYBRID SYSTEM}

Figure 7 shows the difference between the transmitted and received radio signals in the frequency domain. The received signal measured after the BPF and before the electrical amplifier. Subfigure 7(b) clarifies the power reduction of the received signal of the first subcarrier where the central frequency lays at $62 \mathrm{dBm}$. While Subfigure 7(d) shows the received signal of a SCM group with four subcarrier.

To evaluate the performance of the hybrid system a comparison between hybrid RoF WDM with 6 WDM channels and the hybrid RoF SCM/OCDMA/OTDM with 4 MD codes, double weight and 6 time slots has been accomplished in Figure 8. Where the proposed system gives lower performance but much higher scalability. The proposed system which designed with 4 subcarriers, 4 OCDMA codes and 6 OTDM channels can transmit 96 radio signals while only 6 channels in the system of hybrid RoF WDM. Figure 9 shows the eye diagram of the first user of the proposed hybrid RoF SCM/OCDMA/OTDM system with 10 Gbps at 50 Km of distance (SSMF-DCF).

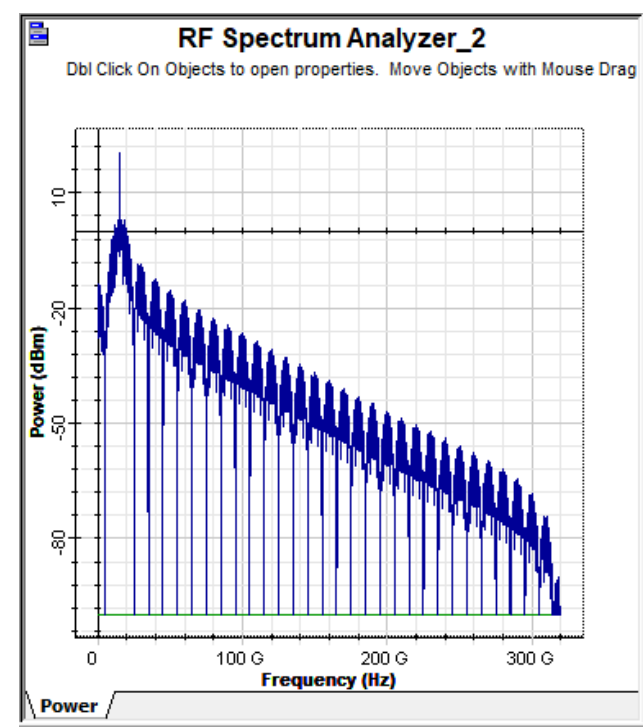

(a) The transmitted signal of a single subcarrier

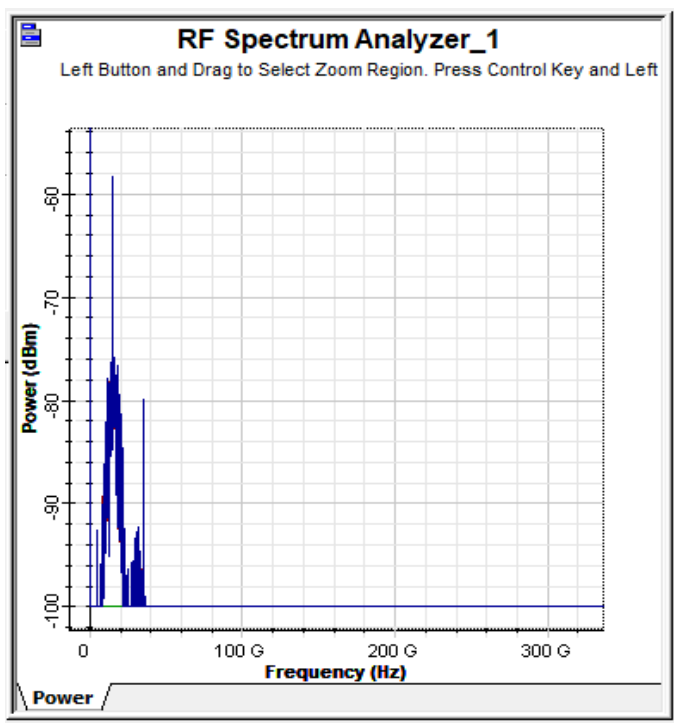

(b) The received signal of a single subcarrier 


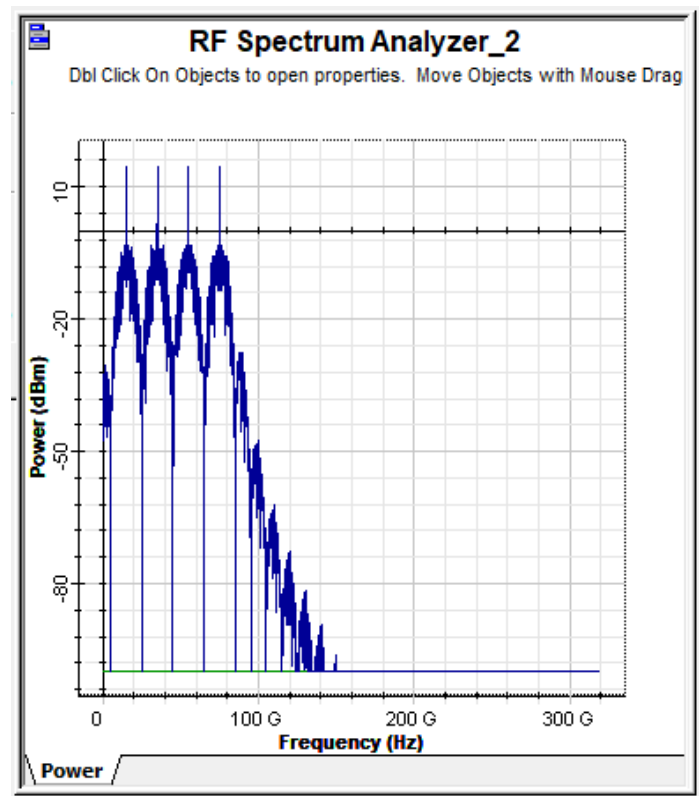

(c) The multiplexed transmitted signal of four subcarrier signals

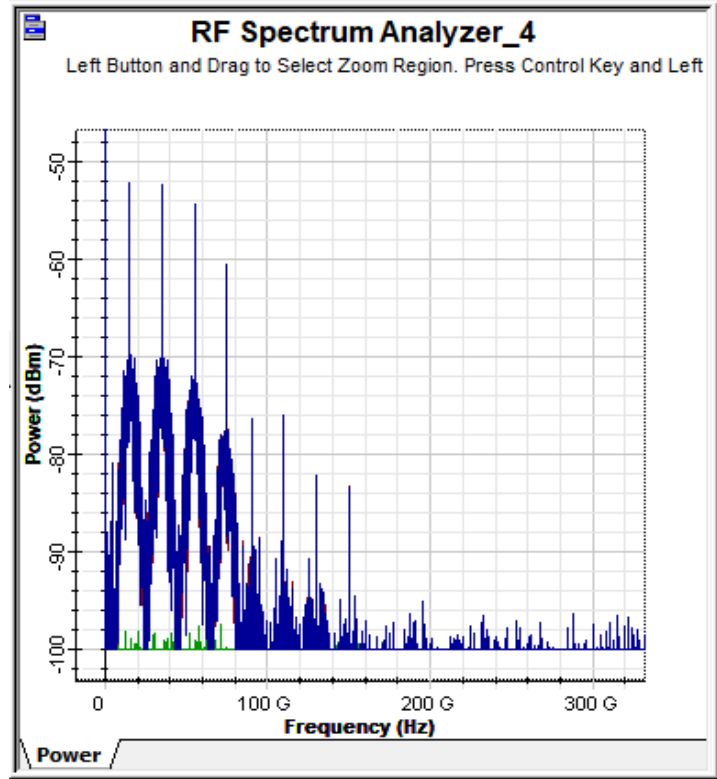

(d) The multiplexed received signal of four subcarrier signals in the frequency domain

Figure 7. difference between the transmitted and received radio signals in the frequency domain

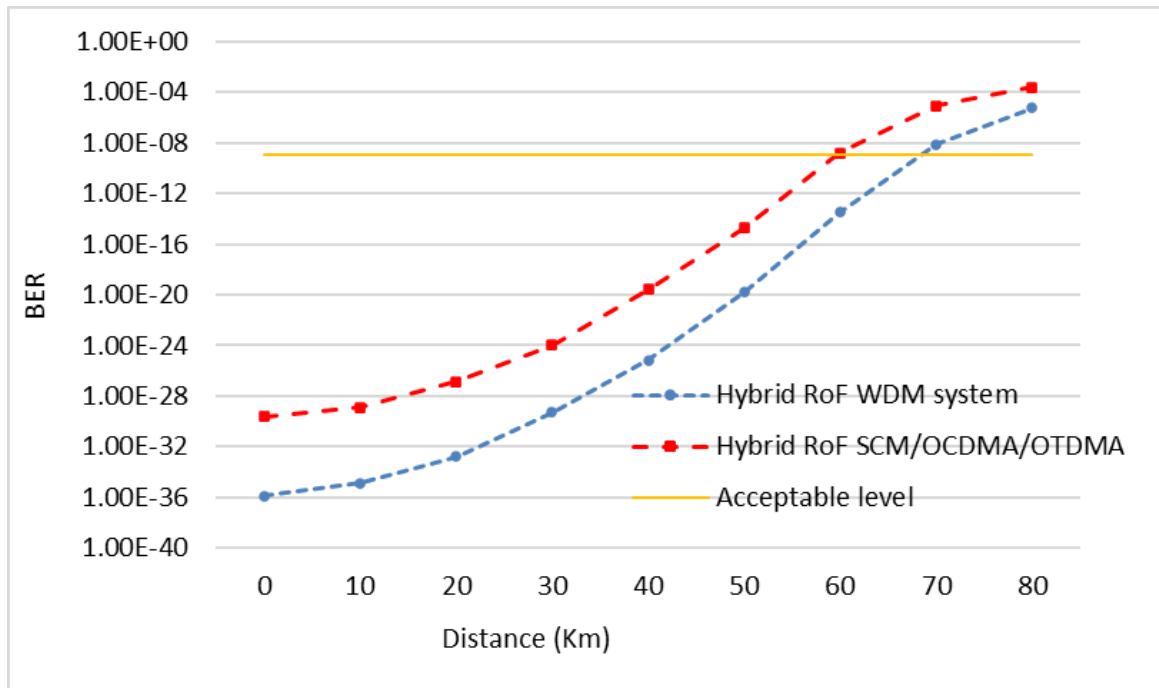

Figure 8. BER versus distance for hybrid RoF WDM with 6 channels and hybrid RoF SCM/OCDMA/OTDM with 4 subcarriers, 4 MD OCDMA codes, double weight and 6 time slots

Figure 10 shows the total supported number of SCM groups of the entire system for M-OCDMA codes of a single OCDMA group over N-channel OTDM system. The OCDMA codes number can be increased by taking into account the limitation of each code type. For example, according to (14) the number maximum users in MD OCDMA with $\mathrm{W}=2$ is 77 and when $\mathrm{W}=4$ is 88 user (at acceptable level BER of $10^{-9}$ ). The other factor of the hybrid system improvement is the number of OTDM channels $\mathrm{N}$, as it is clear in Figure 9, where more channel lead to increase the total number of supported simultaneous users (if the number of users in an OCDMA group is 77 will be doubled with two channels, 308 with four channels and so on). Nevertheless, in the same time the dedicated bandwidth of each user will decrease with this OTDM channel increment. 


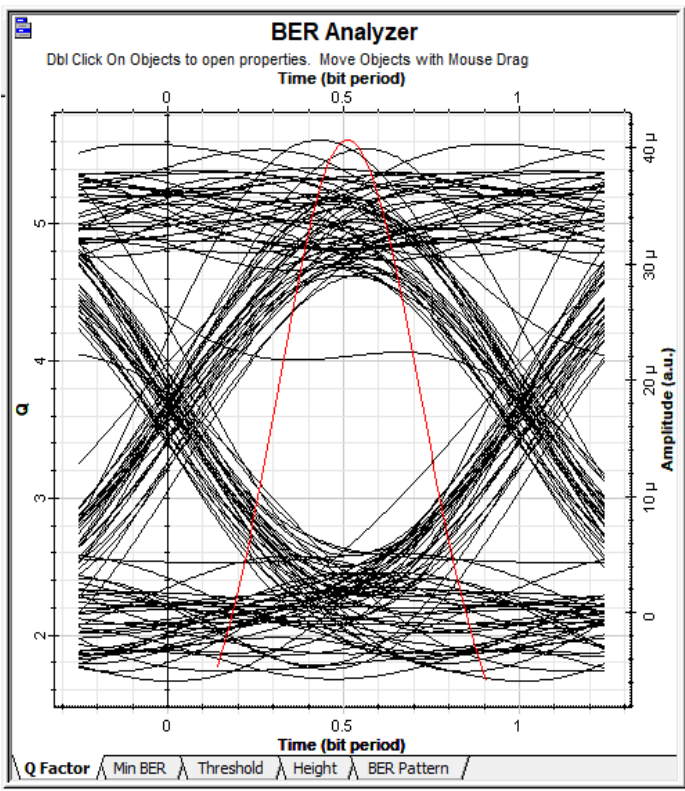

Figure 9. Eye diagram of the hybrid RoF SCM/OCDMA/OTDM at $50 \mathrm{Km}$ of distance

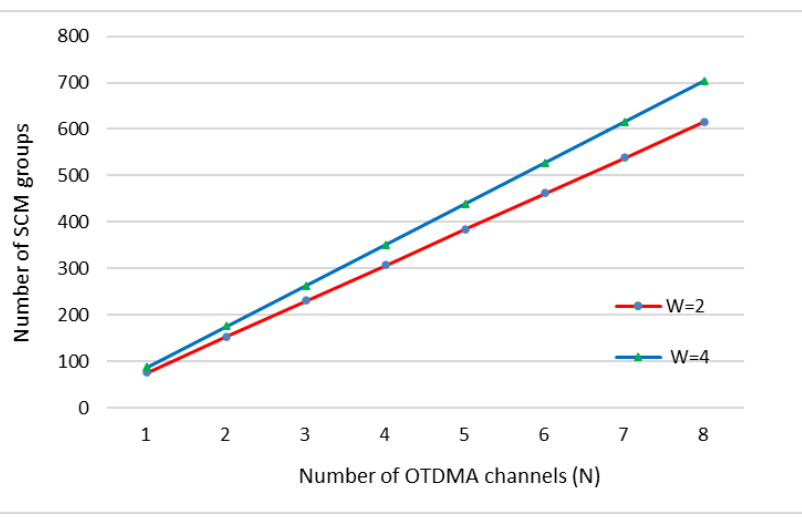

Figure 10. Maximum number of the achievable SCM groups with proposed RoF SCM/OCDMA/OTDMA hybrid system with one dimension MD code

\section{CONCLUSION}

To the best of our knowledge, a new architecture RoF SCM/OCDMA/OTDM hybrid system has been successfully designed and simulated based on MD OCDMA code, which offers an increment in the network scalability while ensuring sufficient data rate and bit error rate. X of SCM signals can be encoded by a single OCDMA code, and M of OCDMA signals can be transmitted in different channels of an OTDM system. The results of the comparison study show that the proposed hybrid system gives an acceptable performance level with a considerable improvement of the scalability compared to RoF WDM hybrid system. Where the proposed system which designed with 4 subcarriers, 4 OCDMA codes and 6 OTDM channels can transmit 96 radio signals while only 6 channels in the system of hybrid RoF WDM. The theoretical results show that more channel of OTDM lead to increase the total number of supported simultaneous users. However, the bandwidth of each user will decrease with this OTDM channel increment.

\section{REFERENCES}

[1] T. S. Rappaport, Y. Xing, G. R. Mac Cartney, Jr., A. F. Molisch, E. Mellios, J. Zhang, " Overview of millimeter wave communications for fifth-generation (5G) wireless networks-with a focus on propagation models, "IEEE Transactions on Antennas and Propagation, vol. 65, no. 12, pp. 6213-6230, 2017.

[2] C. B. M. Rashidi, S. A. Aljunid, A.K. Rahman, M.S. Anuar, and S. Yaakob, "Effective design for optical CDMA based on radio over fiber (RoF) technique, " MATEC Web of Conferences, vol. 97, p. 01100, 2017.

[3] H. Sooyoung, et al, "Theodore S. R., Katsuyuki H., and Jeongho P., Proposal on millimeter-wave channel modeling for 5G cellular system," IEEE Journal of Selected Topics in Signal Processing, vol. 10, no. 3, pp. 454-469, 2016.

[4] C Chen., Z. Chongfu, and Q. Kun, "OCDMA based 60-GHz radio over fiber system for next generation wirele access networks, " 2012 International Conference on Microwave and Millimeter Wave Technology (ICMMT), Shenzhen, pp. 1-4, 2012.

[5] C. Hsu Chih, Y. Chih-Ta, and D. Ing-Jr, "Performance enhancement of optical CDMA by differential-phase method for radio-over-fiber transmissions," Mathematical Problems in Engineering, vol. 2013, p. 901871, 2013.

[6] Z. Ibrahim, et al, "Performance Analysis of Optical CDMA Based on Radio over Fiber (RoF) Technique," 20163 rd International Conference on Electronic Design (ICED), Phuket, pp. 132-135, 2016.

[7] A. O. Aldhaibani, S. Yaakob, R.Q. Shaddad, S.M. Idrusa, "5Gb/s hybrid WDM/TDM PON using radio over fiber technique," Optik, vol. 124, no. 18, pp. 3678-3681. 2013.

[8] D. Wake, A. Nkansah, and J. Gomes," Radio over fiber link design for next generation wireless," Journal of Lightwave Technology, vol. 28, no. 16, pp. 2456-2464, 2010.

[9] C. Zhang, L. Wang, and K. Qiu, "Proposal for all-optical generation of multiplefrequency millimeter-wave signals for RoF system with multiple base stations using FWM in SOA," Optics Express, vol. 19, no. 15, pp. 13957-13962, 2011. 
[10] S. Chaudhary, D. Thakur, and A. Sharma, "10 Gbps-60 GHz RoF transmission system for 5 G applications," Journal of Optical Communications, vol. 11, no. 3, 2017.

[11] Z. Ibrahim, C. B. M. Rashidi, S. A. Aljunid, A. K. Rahman, and M. S Anuar, "Performance evaluation of flexible cross correlation (FCC) OCDMA code based on radio over fiber (RoF) simulation system," Indonesian Journal of Electrical Engineering and Computer Science, vol. 13, no. 2, pp. 543-550, 2019.

[12] K. S. Alaoui, Y. Zouine, and J. Foshi, "Novel configuration of radio over fiber system using a hybrid SACOCDMA/OFDM technique," Advanced Intelligent Systems for Sustainable Development, vol 915, pp 135-144, 2019.

[13] M. Islam, N. Ahmed, S. Ali, S. A. Aljunid, R. B. Ahmad, and M. S. Ali, "Hybrid (OCDMA/WDM) system with dpsk modulation using different detection technique at bit rate $2.5 \mathrm{Gbps}$ for optical access network," International Journal of Microwave and Optical Technology, vol. 11, no. 4, pp. 303-309, 2016.

[14] N. Ahmed, S. A. Aljunid, H. A. Fadil, R. B. Ahmad, and M. A. Rashid, "Hybrid OCDMA over WDM System Using Modified Double Weight (MDW) Code for Optical Access Network,"Advanced Materials Research, vol. 440, no. 2, pp. 3878-3883, 2012

[15] K. S. Alaoui, J. Foshi, and Y. Zouine "Radio over fiber system based on a hybrid link for next generation of optical fiber communication," International Journal of Electrical and Computer Engineering, vol. 9, no. 4, pp. 2571-2577, 2019.

[16] K. A. Memon, A. W. Umrani, M. A. Unar, and W. Shah, "Implementation \& performance analysis of bidirectional FSO channel in hybrid TDM/WDM gigabit passive optical network," 3 C Tecnología. Glosas de innovación aplicadas a la pyme, special issue, pp. 166-181, 2019.

[17] N. Alsowaidi, T. Eltaif, M. R. Mokhtar, and S. A. Qasem, "Design of cost efficient optical code division multiple access (CDMA) channels overlay wavelength division multiplexing (WDM) grid," International Journal on Communications Antenna and Propagation, vol. 9, no. 1, pp. 55-61, 2019.

[18] A. Stok, E.H. Sargent, "The role of optical CDMA in access networks," IEEE Communications Magazine, vol. 40, no. 9 , pp 83-87, 2002.

[19] B.K. Kim, S. Park, Y. Yeon, B.W. Kim, "Radio-over-fiber system using fiber-gratingbased optical CDMA with modified PN codes," IEEE Photonics Technology Letters, vol. 15, no. 10, pp. 1485-1487, 2003.

[20] M. Awad, I. Dayoub, A.O. M'Foubat, J.M. Rouvaen, "The inter-modes mixing effects in mode group diversity multiplexing," Optical Communication, vol. 282, no. 19, pp. 3908-3917, 2009.

[21] F. Baklouti, I. Dayoub, S. Haxha, R. Attia, A. Aggoun, "Novel method for improving the capacity of optical MIMO system using MGDM," IEEE Photonics Journal, vol. 6, no. 6, pp. 1-15, 2014

[22] M. Awad, I. Dayoub, W. Hamouda, J.M. Rouvaen, "Adaptation of the mode group diversity multiplexing technique for radio signal transmission over multimode fiber," IEEE/OSA Journal of Optical Communications and Networking, vol. 3, no. 1, pp. 1-9, 2011.

[23] M. Kasmi, F. Bahloul, and S. Mhatli, "Implementation of an optical GFDM system based on graded-index fiber," Optical Communication, vol. 457, pp. 124738-124744, 2020.

[24] M. Motealleh, and M. Maesoumi, "Simulation of a SAC-OCDMA 10 User x 15 Gb/s System Using MD Code," International Journal of Optics and Applications, vol. 4, no. 1, pp. 20-26, 2014.

[25] A. Cherifi, N. Jellali, M. Najjar, S. A. Aljunid, and B. S. Bouazza, "Development of a novel two-dimensionalSWZCC - Code for spectral/spatial optical CDMA system," Optics and Laser Technology, vol.109, pp. 233-240, 2019. 